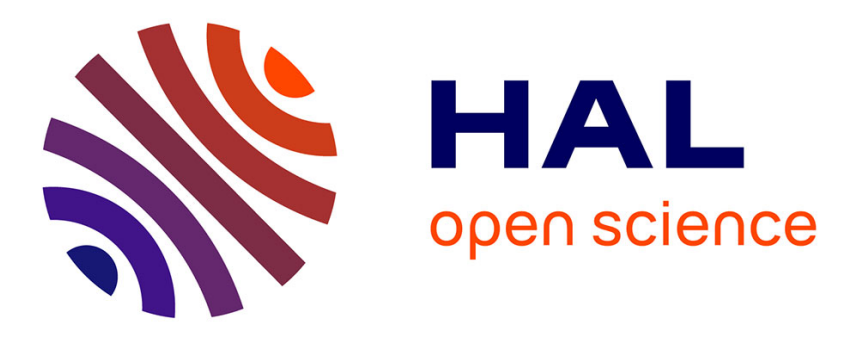

\title{
Implementation of Lean Project Planning: A Knowledge Transfer Perspective
}

Lise Lillebrygfjeld Halse, Kristina Kjersem, Jan Emblemsvåg

\section{To cite this version:}

Lise Lillebrygfjeld Halse, Kristina Kjersem, Jan Emblemsvåg. Implementation of Lean Project Planning: A Knowledge Transfer Perspective. IFIP International Conference on Advances in Production Management Systems (APMS), Sep 2014, Ajaccio, France. pp.248-255, 10.1007/978-3-662-447338_31. hal-01387189

\author{
HAL Id: hal-01387189 \\ https://hal.inria.fr/hal-01387189
}

Submitted on 25 Oct 2016

HAL is a multi-disciplinary open access archive for the deposit and dissemination of scientific research documents, whether they are published or not. The documents may come from teaching and research institutions in France or abroad, or from public or private research centers.
L'archive ouverte pluridisciplinaire HAL, est destinée au dépôt et à la diffusion de documents scientifiques de niveau recherche, publiés ou non, émanant des établissements d'enseignement et de recherche français ou étrangers, des laboratoires publics ou privés.

\section{(c)(1)}

Distributed under a Creative Commons Attribution| 4.0 International License 


\title{
Implementation of Lean Project Planning: A knowledge transfer perspective
}

\author{
Lise Lillebrygfjeld Halse $^{1}$, Kristina Kjersem ${ }^{1}$, Jan Emblemsvåg ${ }^{2}$ \\ ${ }^{1}$ Molde University College, Specialized University in Logistics, Molde, Norway \\ \{lise.l.halse, krisina.kjersem\} @himolde.no \\ ${ }^{2}$ Vard Group AS, Ålesund, Norway \\ jan.emblemsvag@vard.com
}

\begin{abstract}
During the last decades, the Norwegian shipbuilding industry has successfully delivered complex and customized offshore vessels for the global market. This industry has historically been characterized by highly skilled workmanship that makes flexible adaptations and improvements in the production process possible, which has been a competitive advantage of the industry. Nevertheless, more complex projects, competitive pressure and globally distributed value chains, call for more formalized planning and cross learning between shipbuilding projects. Consequently, shipyards in the industry have started to focus more on structured planning tools such as Lean Project Planning (LPP). In this paper, we consider the implementation of LPP from a knowledge transfer perspective, with emphasize on the role of the context in the implementation process.
\end{abstract}

Keywords: Project Planning, Knowledge transfer, Implementation, Lean shipbuilding.

\section{Introduction}

The Norwegian shipbuilding industry delivers complex and customized vessels for offshore purposes using an Engineer-To-Order (ETO) approach [1]. In this kind of production, customers' needs initiate the process of design, engineering and production [2]. Historically, this industry is renowned for highly skilled craftsmanship that makes independent adaptations and improvements in the production process, involving exchange of tacit knowledge [3]. In shipbuilding, project is the norm, and there is a problem with poorly planned and executed project [4]. The reason is claimed to be shortcomings of planning tools that were used earlier.

Consequently, Lean Project Planning (LPP) has been proposed as a new approach in this shipbuilding industry [4]. LPP represents a synthesis of Lean thinking ideas, Earned Value Management approach (EVM) and Last Planner System (LPP). In principle, however, Lean philosophy may not seem to be fully appropriate for ETO producers [5], and in particular manufacturing of low-volume products such as customized ships, which is the focus of this study. Moreover, in a production environment characterized by informal planning and tacit knowledge exchange, the implementation of a formalized planning system may be further complicated, as this necessitates the conversion

adfa, p. 1, 2011.

(C) Springer-Verlag Berlin Heidelberg 2011 
from tacit to explicit knowledge through formalized procedures and statements. Previous research has rarely addressed this issue. We claim that in order to understand the barriers in the implementation of formalized planning systems, we need to view the process as knowledge transfer, where the context of the recipient organization will influence to what extent and in which way the knowledge is absorbed. In this study, the implementation of Lean Project Planning (LPP) in two shipyards is studied, representing two different organizational contexts.

The rest of the paper is organized as follows: First, we present theory related to implementation of management ideas from a knowledge perspective concluding with the need to do studies where the implementation context is addressed. Thereafter, the management idea in this study, LPP, is described. We briefly address the methodology used in this study, and present the research context and the findings, which is separated in two parts: status before implementation of LPP and the implementation into the two research contexts. Finally, we present the discussion, conclusions and contribution of this paper.

\section{$2 \quad$ Literature review}

Implementation of Lean into the manufacturing industry has been considered as a spread of a management idea, which is translated into different organizational contexts [6]. However, such ideas also represent packages of knowledge that aim at improving organizations' performance and competitiveness. This suggests analyzing the implementation of management ideas as transfer and acquisition of knowledge, which is the approach in this paper.

\subsection{Implementation as knowledge transfer}

Generally, implementation of management practices, ideas and tools represents knowledge transfer between organizational units, where translators of the practices and ideas shape knowledge constructs in this process [7]. Several factors may affect how readily knowledge will transfer between organizational units. The nature or form of knowledge is an important aspect, and it has been claimed that explicit knowledge that is embedded in technology is more easily transferred than tacit knowledge not embedded in technology [8]. According to Gupta and Govindarajan Gupta [9], the absorptive capacity of the receiving unit is the most significant factor for successful transfer of knowledge in multinational companies. Here, knowledge inflow depends on the richness of transmission cannels, motivation to acquire knowledge, and capacity to absorb incoming knowledge. Hence, the absorptive capacity concerns the employees' ability and their motivation, and in particular the interaction between these [10]. Moreover, the absorptive capacity is claimed to develop cumulatively, building on the existing knowledge base [11].

Some claim that theoretical arguments and empirical research addressing the individual or interpersonal levels antecedents of absorptive capacity are still lacking in this 
field of research [12]. Furthermore, previous studies have shown that the implementation and outcome of management ideas such as Lean vary considerably across organizations [6, 13]. This implies that the knowledge these kinds of models represents, are 'translated' into a new context, where the recipient organization plays an active role in the adoption of practice and ideas. This will be addressed in the next section.

\subsection{Knowledge transfer and context}

In organizations, people have developed their own particular way of cooperating and sharing knowledge. In line with theory on absorptive capacity, the 'match' between new knowledge and the existing knowledge residing in the organization's social network will facilitate knowledge absorption. From this we would expect that social networks characterized by informal coordination and sharing of tacit knowledge would experience challenges when implementing formalized planning systems, since the existing knowledge base is not articulated, making it difficult to build on further by new and explicit knowledge. This dimension of knowledge acquisition can also be understood from a motivational point of view, as people will be motivated to acquire knowledge that fits their existing working practice and knowledge [10]. Hence, the motivation to acquire knowledge, and thus the absorptive capacity, is dependent on the social context.

This study addresses the gap in the literature regarding contextualization of absorptive capacity, and in particular, the translation of management practices and ideas, where previous studies have been at a higher level of abstraction. We contribute filling the gap by providing a case study of implementation of Lean Project Planning (LPP) in an ETO shipbuilding environment. The difference in context is addressed by studying the implementation of LPP in two shipyards. In the following, we will briefly describe LPP, which represents the knowledge that is sought introduced and implemented at these shipyards.

\subsection{Lean project planning (LPP)}

LPP is based on Lean thinking [14] adapted to construction industry (Lean Construction) including elements from Last Planner System (LPS) [15] and Earned Value Management (EVM) [16].

Lean Thinking is represented here by Plan Do Check Act (PDCA) cycle, a basic problem-solving approach, which in the LPP context involves making problems visible, finding proper solutions, checking the results and acting on deviations [17].

Lean Construction applies production-based ideas from lean thinking to project delivery within construction industry [18]. In such projects, lean changes the way project is managed during the building process. Lean Construction extends from the objectives of a lean production system - maximize value and minimize waste - to specific techniques, and applies them in a new project delivery process. Lean Construction is particularly useful on complex, uncertain and quick projects. It challenges the belief that there must always be trade-offs between time, cost, and quality. Since the 1990s, 
lean construction community recognized the need for a change in the way traditional project management plan and measure activities in a project.

LPS is a planning tool intended to increase planning reliability by decreasing workflow variability, through recognizing and removing activity constraints, identifying root causes for non-completion of plans and monitoring its improvement by means of Percent Plan Complete (PPC) [19]. LPS is a way to involve the 'last planner' - the last link in the decision chain - within the planning process by making 'promises' and then measure the percent of completed activities out of the promised ones (PPC) [20].

EVM is a technique used within the planning system for measuring the progress of a project by comparing the baseline of the project with reported physical results, the resources consumed and the remaining hours to the completion per activity [16]. A good performance metrics used by EVM is the Cost Performance Index (CPI) used to calculate and predict cost at completion of the project within a finite range of values after only 15-20 per cent completion of the project [21].

Each of the elements above has an important role in the LPP. PDCA involves identifying problems, find solutions and check the results. In LPP this is done in weekly meetings, starting with checking the status of previous week's planned activities. LPS is central for involving people within the planning process in order to achieve reliable promises and identifying problems in the near future. Important here is to involve the last link in the decision process, e.g. foremen as well as facilitating good communication and information flow between project participants. EVM contributes to making visible the progress and earned value at any stages during the building process.

\section{Methodology}

In this study, we aim at contributing to filling the gaps in the literature by studying the implementation of LPP in an ETO production environment, and where the differences in context are addressed. Despite having thorough knowledge of the LPP tool, the implementation of the tool is explorative in its nature, making a case study design appropriate [22]. Two shipyards are chosen as cases in this study: shipyard A and B. These shipyards were originally separate companies that were acquired by a larger group in the 1990s. They have, however, operated to a large extent as separate entities where most activities as design, engineering and production were carried out locally. Several years ago, the shipbuilding group started a centralization process by taking electro, design, piping, and accommodation departments outside shipyards and recreated them as distinct entities. This reorganization, together with the production of the hull outside Norway, created new challenges for the planning process. The present study involves a comparative research setting where the implementation process at the two shipyards is compared in order to find the relationship between barriers and the success of implementation.

Data is collected through participation in meetings and in-depth semi structured interviews employees in the head office and the two shipyards (three sites). Internal reports, minutes of meetings, and presentations constitutes additional data sources. Fur- 
thermore, one of the authors is the founder of LPP and has been involved in the implementation process at shipyard A. The interviews and the document study were aimed at revealing what parts of the LPP was sought implemented, how the implementation process was carried out and what kind of obstacles was experienced in the process. Furthermore, the planning systems and processes used before the implementation were mapped. Finally, the respondents were asked to reflect upon the reasons for the difficulties they experienced in the process.

\section{Context - the situation before implementation}

One of the most important characteristic of Norwegian shipbuilding industry is its flexibility, which gives customers the possibility to decide many features of the vessel quite late during the building process [23]. Maintaining this flexibility while dealing with increased complexity of vessels, competition on shorter delivery periods, and lower building prices, involves a constant focus on improving all processes in the building projects. The whole shipbuilding process is performed through a concurrent engineering approach, which implies that production of any part of the vessel can be started while the engineering phase is still being in progress. In concurrent shipbuilding, engineering, procurement and production activities are performed in near concurrency throughout the project execution - in contrast to the sequential approach where the vessel is engineered, equipment is ordered and then start the production process. The concurrent process, the participation of several different entities (internal and external), and the geographically distributed value chain (hull production outside Norway) represent demanding conditions for the implementation of a reliable planning system.

In this study the emphasis is on the planning process for the outfitting phase of the project. The outfitting phase is defined as the stage in which some of the last components are mounted on board, before the vessel testing phase. The number of participants during the outfitting phase in each project varies from 200-700 workers belonging to different specializations (steel workers, piping, accommodation, electro, etc.).

Before the LPP implementation, both shipyards were generally able to meet the requirements regarding cost and delivery times. This can be traced back to the long experience of the production workers, who were able to make prompt decisions based on their experience and tacit knowledge. Both shipyards were using formalized planning systems only to a moderate extent. In particular, the participation of people from production in the planning process was limited or absent, while a dedicated person, the Planner, created the plans based on data from previous projects. Furthermore, there were no recordkeeping of deviations in order to avoid recurrence of mistakes at both shipyards. In both shipyards, the communication flow took place mainly through informal channels.

However, there were some differences between the two shipyards regarding the structure and formalism of meetings. Shipyard B had before the implementation regular planning meetings on a weekly basis. From these meetings Minutes of meetings (MOM) were written by the Planner and sent to designated persons within the project. Such structure in meetings was not present at Shipyard A, where the meetings were 
more informally organized, and where MOM was seldom written. Furthermore, in shipyard B there existed a planning tool to follow the evolution of the projects in terms of resources. The diagrams were however based on the Planner's experience. In Shipyard A there was lack of formal control over the projects regarding resources and time, and decisions were made based on people's experience.

\section{Implementation into two different production contexts}

Demands of shorter building periods, organizational changes (centralization), and coordinating challenges due to the distributed production process (foreign hull producing shipyard and local shipyard for outfitting), motivated these shipyards to look into their planning processes. LPP was implemented and adapted with the purpose of enhancing communication within the planning process [23]. This was done by standardizing the meeting structure, providing physical progress measurement, and proactively eliminating barriers that could affect completion of any activity. Another purpose for implementing this tool was to achieve commitment to the project plans by involving people executing the activities within the planning process. Shipyard A succeeded in implementing the most important aspects of LPP as described above. Participation was achieved by asking foremen to prepare week plans with activities that can be executed by own team during the next period, so that they were committed to the plan and felt responsibility to complete activities as planned. Furthermore, Discipline Coordinators (DC's) were formally invited to weekly meetings, and the meetings became more structured and formalized. Moreover, deviations became recorded systemically on the week plan and followed up by designated people. In general, communication flow in projects was improved, as more people were involved in the planning process, as well as increased commitment through written plans. The results of implementing this tool at Shipyard A were very good, which led the management of the shipbuilding group to encourage the other shipyards to implement LPP.

The second shipyard in this study, Shipyard B, approached the LPP differently than shipyard A. After being introduced to the planning tool, they tried it for a few months and then decided that it is not fitted for their way of working. Instead, they reinforced the usual meeting procedure with some small adjustments: The General Plan was updated based in the reporting from own Discipline (DCs) and presented during the weekly meeting. Furthermore, an adapted EVM version was introduced. However, this was adapted only at a general plan level, not at a lower level in the project organization. Moreover, deviation was only discussed and solved in connection with formal, however mostly in informal meetings, but there were no records made in order to prevent reoccurrence of the deviations.

\section{Discussion and conclusions}

The purpose of this paper was to study the implementation of LPP in the shipbuilding industry from a knowledge perspective. In particular, we wanted to analyze the role of 
context in the implementation process, which has been done by studying the implementation of LPP at two separate shipyards in the same shipbuilding group.

We found that Shipyard B had somewhat more formalized planning routines than Shipyard A before implementation of LPP. Hence, we should expect that the knowledge base of Shipyard B should be more adapted to the new knowledge represented by LPP. However, this study finds that this has not been the case. On the contrary, the implementation of LPP has so far been more successful at Shipyard A. Literature on absorptive capacity emphasize the importance of interaction between the knowledge base and employees' motivation [11]. In our study, we found that there were differences regarding motivation at the management level. In shipyard A, the manager participated actively in developing LPP and implementing the planning tool at the shipyard. At Shipyard B, the managerial level found that the existing planning tool functioned satisfactory, and hence were not motivated to implement a new and more formalized tool. This finding illustrates the importance of the interaction between both knowledge and motivation for a successful implementation. Furthermore, the lack of formalized planning systems at Shipyard A could be considered as an advantage when implementing a new planning tool, as this organization may be more open to new ways for organizing working processes. Finally, our findings may indicate that these two shipyards despite of being a part of the same shipbuilding group residing in a cluster characterized by shared norms for knowledge sharing and extensive use of informal coordination [24], represent two different organization cultures. Based on preliminary findings, we can deduce that the organizational culture at Shipyard A emerges as more open to external impulses than at Shipyard B. The role of the organizational culture should be addressed in further studies. Furthermore, as this study is carried out in a relatively early stage of the implementation process, data should be collected at later stages in the implementation process, preferably including other shipyards in the group implementing LPP.

\section{References}

1. Haartveit, D.G.,Semini, M., Alfnes, E.: Integration Alternatives for Ship Designers and Shipyards, In, Frick, J., Laugen, B., (eds.) Advances in Production Management Systems. Value Networks: Innovation, Technologies, and Management. pp. 309-316. Springer Berlin Heidelberg (2012)

2. Hicks, C.,McGovern, T., Earl, C.F.: A Typology of UK Engineer-to-Order Companies. International Journal of Logistics: research and applications. 4(1), 43-56 (2001)

3. Asheim, B., T., Isaksen, A.: Regional Innovation Systems: The Integration of Local 'Sticky' and Global 'Ubiquitous' Knowledge. Journal of Technology Transfer. 27(1), 77-86 (2002)

4. Emblemsvåg, J.: Lean Project Planning in Shipbuilding. Journal of Ship Production and Design. 30(1), 1-10 (2014)

5. Cooney, R.: Is "lean" a universal production system? Batch production in the automotive industry. International Journal of Operations \& Production Management. 22(9/10), 11301147 (2002)

6. Morris, T., Lancaster, Z.: Translating Management Ideas. Organization Studies. 27(2), 207233 (2006) 
7. Sahlin-Andersson, K., Engwall, L.: Introduction, In, Sahlin-Andersson, K., Engwall, L., (eds.) The Expansion of Management Knowledge. Carriers, flows, and sources. Standford University Press, Standford, CA (2002)

8. Zander, U., Kogut, B.: Knowledge and the Speed of the Transfer and Imitation of Organizational Capabilities: An Empirical Test. Organization Science. 6(1), 76-92 (1995)

9. Gupta, A.K., Govindarajan, V.: Knowledge flows within multinational corporations. Strategic Management Journal. 21(4), 473-473 (2000)

10. Minbaeva, D., et al.: MNC knowledge transfer, subsidiary absorptive capacity, and HRM. Journal of International Business Studies. 34(6), 586 (2003)

11. Cohen, W.M., Levinthal, D.A.: Absorptive Capacity: A New Perspective On Learning And Innovation. Administrative Science Quarterly. 35(1), 128-152 (1990)

12. Minbaeva, D.B., et al.: A retrospective on: MNC knowledge transfer, subsidiary absorptive capacity, and HRM. Journal of International Business Studies. 45(1), 52-62 (2014)

13. Giroux, H., Taylor, J.R.: The Justification of Knowledge: Tracking the Translations of Quality. Management Learning. 33(4), 497-517 (2002)

14. Womack, J.P., Jones, D.T.: Lean thinking: banish waste and create wealth in your corporation. Free Press. New York (2003)

15. Ballard, H.G., The last planner system of production control, In: Faculty of Engineering. University of Birmingham: Birmingham (2000)

16. Sumara, J., Goodpasture, J. Earned value-the next generation-a practical application for commercial projects. In: Project Managment Institute 28th annual Seminars \& Symposium. Chicago. (1997)

17. Sobek, D.K., Smalley, A.: Understanding A3 thinking: a critical component of Toyota's PDCA management system. CRC Press. Boca Raton (2008)

18. Koskela, L., Application of the New Production Philosophy to Contruction, In: Technical Report. CIFE, Standford University (1992)

19. Ballard, G., Howell, G. An update onlast planner. In: IGLC. Virgina, USA. (2003)

20. Olano, R.M.,Alarcon, L., Razuri, C. Understanding the relationship between planning reliability and and Schedule performance: A case study. In: IGLC-17. Taipei, Taiwan. (2009)

21. Flemming, Q.W., Koppelman, J.M.: Using Earned Value Management. Cost Engineering. 44(9), 32-36 (2002)

22. Yin, R.K.: Case study research: design and methods. Sage. Los Angeles (2009)

23. Semini, M., et al.: Strategies for customized shipbuilding with different customer order decoupling points. Proceedings of the Institution of Mechanical Engineers, Part M: Journal of Engineering for the Maritime Environment, (2014)

24. Halse, L.L., Governance and transformation of clusters: theoretical models and the case of the maritime cluster in Mid-West Norway, In: 18th EurOMA Conference. Cambridge, UK (2011) 